Departamento de Educación Médica, Facultad de Medicina, Universidad de Concepción. aPsicóloga, Magíster en

Ciencias de la Educación,

mención Didáctica e Innovación Pedagógica.

'Psicólogo, Magíster en Psicología con mención en Psicología Educativa.

'Matrona, Magíster en Evaluación Curricular.

¿Bioquímica, Magíster en Educación Médica para las Ciencias de la Salud. eKinesiólogo, Magíster en Educación Médica para las Ciencias de la Salud.

Financiamiento: Proyecto DEM 2012/03.

Recibido el 8 de agosto de 2014, aceptado el 6 de octubre de 2014.

Correspondencia a: Javiera Ortega B. Departamento de Educación Médica, Facultad de Medicina, Universidad de Concepción, Barrio Universitario $s / n$, Concepción, Chile. Teléfono: 41-2204927 javieraortega@udec.cl

\section{Evaluación de componentes del currículum oculto en estudiantes de medicina}

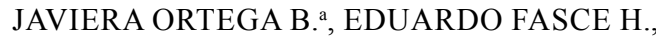 \\ CRISTHIAN PÉREZ V. ${ }^{b}$, PILAR IBÁÑEZ G. ${ }^{c}$, \\ CAROLINA MÁRQUEZ U. ${ }^{\mathrm{d}}$, PAULA PARRA P. ${ }^{\mathrm{e}}$
}

\section{Assessment of hidden curriculum components by medical students}

\begin{abstract}
Background: Hidden curriculum refers to the unwritten, unofficial, and often unintended lessons, values, and perspectives that students learn at the university, which influences the acquisition of professional skills. Aim: To analyze the perception about the influence of the hidden curriculum in the education of medical students at the Universidad de Concepción, Chile. Material and Methods: Qualitative investigation with case study approach. Seventeen graduated medical students were selected by probability sampling. A semi-structured interview was used to collect the information and a content analysis was applied. Results: Forty seven percent of participants recognized having fulfilled their academic expectations. As favorable factors for academic achievement the students underlined clinical practice, access to patients and to clinical fields. As negative factors, they identified the lack of commitment, educational support and overspecialization of their mentors. Conclusions: The results show the strengths and weaknesses of the educational environment of undergraduated medical students. This information should be used to modify teaching environments.
\end{abstract}

(Rev Med Chile 2014; 142: 1452-1457)

Key words: Currículum; Education, medical; Qualitative research; Students, medical.
4 l término "currículum oculto", también denominado "tácito", "latente" y "no escrito", ha sido objeto de diversas definiciones que hacen de este constructo un importante objeto de estudio. De hecho, existen diversas razones para entender el efecto que ejerce el currículum oculto en el ambiente educativo ${ }^{1,2}$. Turbes ${ }^{3}$ lo define como aquellos "mensajes comunicacionales que se establecen en las interacciones interpersonales entre docentes y alumnos, tanto en ambientes clínicos como en otros escenarios". Según Cribb y Bignol ${ }^{4}$, corresponde a "procesos, presiones $y$ fuerzas ajenas al currículum formal, las que a menudo están desarticuladas e inexploradas". Sin embargo, aun cuando el currículum oculto es una dimensión importante en el proceso formativo, la identificación de sus componentes aún no han sido incluidos en los estándares de evaluación ${ }^{5}$, a pesar de que la educación médica es reconocida como un proceso cultural y, por tanto, sujeto a la influencia de fuerzas externas y a problemas derivados de la integración interna ${ }^{6}$. Las señales del currículum oculto provienen de los diferentes ambientes donde se desenvuelve el estudiante y pueden determinar efectos positivos o negativos. Entre estos últimos, se incluyen la pérdida en la credibilidad de los docentes, la pérdida del altruismo, el socavamiento de las conductas éticas ${ }^{7}$ en diversos períodos de la carrera, como es la transición al ciclo clínico ${ }^{8}$. 
Dada la importancia que se otorga a la influencia del currículum oculto en el proceso formativo, nos propusimos indagar las características de sus componentes en estudiantes de medicina chilenos.

\section{Material y Método}

Se utilizó una metodología cualitativa tomando como enfoque el estudio de casos con el propósito de analizar las particularidades y las unicidades que caracterizan a los participantes del estudio, lo cual permite recoger aquello que piensan, sienten y la forma en que actúan'.

Participaron 17 egresados de la carrera de medicina de la Universidad de Concepción (10 hombres y 7 mujeres), al momento de haber rendido el Examen Médico Nacional. La selección fue por muestreo por cuotas, considerando un número de participantes que reuniera aquellas condiciones necesarias para la investigación ${ }^{10}$ hasta haber logrado la saturación de datos. Las técnicas de recolección de información fueron las notas de campo y la entrevista semi-estructurada, la cual cuenta con temas y subtemas que actúan como marco de referencia para el entrevistador ${ }^{9}$. Para su diseño se adaptó el formato de entrevista propuesto por Lempp \& Seale ${ }^{11}$, incluyendo aspectos como cumplimiento de expectativas personales, fortalezas y debilidades del proceso formativo y apreciación de las relaciones profesionales. Las entrevistas se realizaron en horarios y espacios acordados con los egresados con una duración de aproximadamente $60 \mathrm{~min}$, previo consentimiento informado.

A partir de los datos obtenidos, se realizó un análisis de contenido basado en la propuesta de Bardín ${ }^{12}$, quien definió tres etapas fundamentales: preanálisis: selección y análisis de documentos para delimitar las unidades de texto, unidades de análisis o unidades de codificación, como es el caso de frases; explotación del material: identificación de las categorías, las cuales, según Berelson ${ }^{13}$, deben ser homogéneas, exhaustivas (agotar la totalidad del texto), exclusivas (no se debe incluir un mismo elemento en dos categorías diferentes), objetivas (dos codificadores deben llegar a los mismos resultados) y pertinentes (deben adecuarse al contenido y al objetivo); tratamiento e interpretación de los resultados obtenidos: síntesis y selección de los resultados, inferencias e interpretación.

\section{Resultados}

Surgieron tres categorías asociadas al rol que cumple el currículum oculto en el proceso formativo de estudiantes de medicina: percepción sobre el proceso formativo, equilibrio entre la técnica y el cuidado de los pacientes y percepción de sí mismo a lo largo de su trayectoria académica.

\section{Percepción sobre el proceso formativo}

Esta categoría hace alusión a cómo los estudiantes interpretan y dan significado al proceso formativo durante sus estudios, comprobándose que está fuertemente marcado por sus expectativas iniciales: quienes declaraban que éstas no eran claras al comienzo de sus estudios, no lograban realizar una evaluación bien definida sobre la formación recibida. Al contrario, quienes reportaron estar satisfechos con su proceso formativo y declararon sentirse identificados con la carrera, fueron quienes tenían claridad en sus expectativas desde un comienzo. Es así como reconocen haber logrado desarrollar habilidades necesarias para su ejercicio profesional, e identificar sus propias competencias, en especial en la atención de pacientes. En relación a la percepción de los egresados sobre el proceso formativo se identificaron aspectos satisfactorios e insatisfactorios.

\subsection{Percepción sobre los aspectos satisfactorios de la formación}

Hacen mención a los factores académicos y no académicos, dentro de los cuales incluyen un adecuado diseño curricular, la disponibilidad de medios bibliográficos, los campos clínicos con acceso a un gran número de pacientes y disponer de una guía docente:

“...la carrera está bien estructurada, ofrece campos clínicos que facilitan la interacción con un alto número de pacientes $y$, en la mayor parte de las veces, se dispone de guías directas por docentes calificados..." (XIV 2).

Identifican logros asociados a las áreas procedimentales y actitudinales. Entre los primeros destacan los aprendizajes en el abordaje clínico, en las urgencias médicas, y en un manejo integral, condicionante de relaciones empáticas y adaptables a las diferencias culturales. Además, incluyen la concepción de trabajo en equipo, la posibilidad de dirigir una sala de hospital y de poder cooperar 
en la enseñanza de los cursos inferiores. En el área actitudinal, enfatizan la posibilidad de desarrollarse como personas y adquirir habilidades de comunicación, y evalúan como enriquecedora la responsabilidad que tienen como internos:

"...la gran diversidad cultural de los pacientes permite desarrollar habilidades de comunicación acordes con esas diferencias..." (VII 1).

“...un aspecto especialmente positivo para mí fue darme cuenta que era capaz de empatizar con los pacientes y con todo el equipo de trabajo..." (XV 2).

“...poder contribuir al aprendizaje clínico de alumnos de cursos inferiores fue una experiencia enriquecedora..." (X 1).

Otros estudiantes destacan su satisfacción por el trabajo realizado y el reconocimiento que recibieron por éste a través de la respuesta favorable de los pacientes y de los familiares:

"...fue muy motivante recibir el agradecimiento de los pacientes por la atención brindada como también de sus familiares...” (I 2).

Destacan aquellas asignaturas donde percibieron una buena relación estudiante-docente descrita como una relación óptima y horizontal:

“...la mejor relación con mis docentes la obtuve durante el primer año en las tutorías de aprendizaje basado en problemas, donde el clima de trabajo fue muy cálido y estimulante..." (IX 2).

\subsection{Percepción de los aspectos insatisfactorios de la formación}

El proceso de enseñanza es estimado como poco integrador y muy especializado, careciendo de otros aspectos relevantes para la formación médica, ya sea aspectos actitudinales o materias complementarias:

“... la mayor parte de las materias está a cargo de especialistas quienes tienden a ahondar en aspectos propios de su especialidad y dejan poco espacio para abordar otros contenidos de mi interés y no se pone mayor atención a aspectos conductuales...” (XI 2).

Refieren que en algunos años de la carrera existe una inadecuada distribución del tiempo con exceso de teoría y carencia de discusión de casos clínicos. Al respecto, proponen la realización de seminarios, metodología de $\mathrm{ABP}$ o actividades prácticas:

“...hay semestres muy recargados de materia con respecto a otros, especialmente en ramos pre-clínicos. En clinica se echa de menos un mayor número de análisis de casos. Sería bueno utilizar resolución de problemas, tal como se realizaba en primer año..." (XI 2).

Con relación a la actividad docente, algunos estudiantes señalan que en algunas disciplinas clínicas percibieron falta de compromiso y guía:

“...en algunas asignaturas clínicas falta apoyo de parte de los docentes y uno se encuentra un tanto solo..." (I 2).

Con respecto al trato recibido de parte de sus docentes, estiman que se suelen presentar relaciones asimétricas y ser objeto de descalificaciones:

“...en una de mis rotaciones de hospital hubo docentes que me trataron de ignorante frente a los pacientes y el personal de sala: me sentí humillado..." (IX 2).

“...algunas interrogaciones orales fueron muy traumáticas, provocándome temor al momento de enfrentarlas..." (VII 2).

“...cuando uno se siente maltratado por algunos docentes se cuestiona el continuar en la carrera..." (IV 4).

\section{Equilibrio entre la técnica y el cuidado de los pacientes}

Señalan que el adecuado equilibrio entre competencias profesionales de sus docentes y el interés por los pacientes está mediado más por características individuales que por una política institucional.

“... lograr interesarse en la parte humana de los enfermos depende de cada alumno sin que se observe un compromiso generalizado por parte de los médicos..." (XV 2).

“...existen muchos profesionales que demuestran gran vocación, pero también algunos no se ven comprometidos con la atención de sus pacientes..." (III 2).

Señalan que en general no se aprovechan las oportunidades en sala, por escasa presencia de 
docentes. De manera alternativa, han visualizado que los asistenciales cumplen un importante rol formador:

“...los médicos asistenciales disponen de mayor tiempo para estar con uno en las salas mientras que los docentes, quizá si porque tienen muchas ocupaciones, nos dedican menos tiempo..."(VII 2).

Al consultarles sobre características de sus docentes que fueron objeto de admiración, destacan aquéllos que exhiben cualidades humanas y personales $(30 \%)$, alto nivel de competencia profesional $(26,7 \%)$, interés en el aprendizaje de sus alumnos (20\%), preocupación por sus pacientes $(16,7 \%)$ y conducta ética $(6,6 \%)$ :

“...hubo docentes que causaron mi admiración por demostrar un gran interés en dar solución a los problemas de los pacientes y por su intachable conducta ética..." (V 4).

“...siempre admiré a quienes demostraron sólidos conocimientos permitiendo dar solución a los problemas de salud de los enfermos..." (XVI 4).

“...destaco a docentes muy empáticos, con muy buenas relaciones interpersonales, interés en sus alumnos y en sus pacientes...” (I 4).

Sin embargo, también señalan visiones negativas, especialmente falta de interés en la enseñanza, descalificaciones entre pares, carencia de humanismo y trato inadecuado a los alumnos:

“...en algunas rotaciones hubo docentes que ejercieron una influencia negativa por su tendencia a cuestionar decisiones de otros profesionales mediante descalificaciones..." (IV 2).

“...en ocasiones sentí que no me trataban como persona, sólo me transmitían materia, a veces de manera irrespetuosa..." (XII 2).

\section{Percepción de sí mismo a lo largo de su trayec- toria académica}

Los estudiantes mencionan diversos aspectos que han cambiado en ellos debido a la influencia de su proceso formativo. Se refieren a cambios en el aprendizaje, en su vida personal y en su visión del mundo. En cuanto a los cambios en el aprendizaje, informan que han debido optimizar la distribución del tiempo, priorizando sus actividades y adaptando el horario del sueño. Luego adquieren un mayor sentido de responsabilidad al tener contacto con pacientes:

“...se debe optimizar el uso del tiempo, ya sea para establecer las prioridades en las materias de estudio como para aprovechar el tiempo con otros..." (VI 3).

“...la relación con los pacientes estimula el interés por aprender, determina que uno sea más responsable..." (IV 3).

Los principales cambios asociados a su vida personal se relacionan con abandonar gran parte de las actividades recreativas previas; como ir al cine, leer, andar en bicicleta, realizar deportes, tocar algún instrumento musical, escribir; como también reconocer sus limitaciones, y manejar las emociones.

“...la intensidad de los estudios obliga a dejar cosas que hacían bien para el alma... dejar iniciativas personales...” (III 3).

“...la cercanía con las personas me permitió dimensionar el contexto social en que viven, sus carencias y la complejidad de disponer de recursos suficientes para satisfacer tanta demanda..." (II 3).

Por último, reconocen el impacto de enfrentar de cerca la muerte, su afrontamiento y la comunicación con la familia de los fallecidos, las dimensiones del dolor, y comprender el contexto social de las personas al observar las dimensiones de la pobreza, las diversas carencias, las limitaciones presupuestarias que existen en la atención:

“...vivir de cerca la muerte ha sido una experiencia dura y compleja como también enfrentar a la familia. Sin embargo, son realidades que debemos aprender a afrontar y que nos hace más fuertes..." (X 3 ).

\section{Discusión}

El currículum oculto es una variable fundamental que incide en el proceso formativo de los estudiantes y por el valor que ésta ejerce en el aprendizaje debería estar incorporada en los estándares de evaluación ${ }^{5}$. Nuestros resultados son semejantes a investigaciones realizadas en otras escuelas de medicina y un elemento que resalta es el tipo de relación que se puede establecer entre 
docentes y estudiantes, relación a veces basada en la humillación y el mal trato. Phillips y Clarke ${ }^{14}$ utilizan un cuestionario electrónico en estudiantes de tres escuelas médicas de Canadá, detectando procesos de discriminación sobre pacientes y descalificación entre médicos de diferentes especialidades, determinando en los alumnos un replanteamiento de sus valores y de sus creencias iniciales. Asimismo, en Harvard ${ }^{15}$ utilizan reflexiones escritas de estudiantes de tercer año, identificando nueve temas centrales del currículum oculto, que incluyen el abuso del poder y de la jerarquía de los médicos, deshumanización de la práctica médica, criterios arbitrarios de evaluación, supresión de las respuestas emocionales frente a las vivencias del sufrimiento y la muerte, carencia de acciones médicas enfocadas a la integridad del paciente y necesidad de desarrollar virtudes profesionales, aspectos coincidentes con aquellos obtenidos en nuestro estudio.

Teniendo mayor información al respecto, se podría llegar a proponer un rol del docente que se asemeje al perfil profesional que cada carrera tiene. Al respecto, Thiedke y cols. ${ }^{16}$ aplicaron a 119 estudiantes un cuestionario destinado a evaluar comportamiento del médico con el paciente, encontrando bajos puntaje en la visión del rol profesional, en la entrega de información sobre su enfermedad y tratamiento, y en aplicar las técnicas de entrevista aprendidas en clases. Wong y Trollope-Kumar ${ }^{17}$ analizan 604 narraciones de 65 estudiantes de medicina de McMaster, comprobando la influencia positiva o negativa del rol de modelos, la visión de dilemas éticos, las aprensiones personales frente al sufrimiento de pacientes y la carencia de virtudes profesionales. Por otra parte, Neumann y cols. ${ }^{18}$ revisaron 18 estudios sobre niveles de empatía en alumnos de medicina comprobando su significativa disminución a lo largo de los años de estudio. Entre los posibles factores causales detectados destacan situaciones de humillación, intimidación y discriminación. También describen en los estudiantes la pérdida del entusiasmo e idealismo que presentaban al inicio de sus estudios al enfrentarse a realidades clínicas como el sufrimiento y la muerte, además de una visión negativa del rol de modelos.

En consideración a la importancia del currículum oculto en la formación de estudiantes de medicina, queremos insistir en la necesidad de generar políticas institucionales para resguardar un ambiente educativo favorecedor del desarrollo académico con una evaluación constante del currículum oculto en la implementación de la nueva currícula.

Los resultados presentados son parte de una primera aproximación orientada a identificar componentes del currículum oculto. Es fundamental continuar líneas de investigación que propendan a analizar los factores académicos y personales que influyen en la conformación de un currículum oculto como el aquí descrito.

\section{Referencias}

1. Callaghan A. Emotional congruence in learning and health encounters in medicine: addressing an aspect of the hidden curriculum. Adv in Health Sci Educ 2013; 18: 305-17.

2. Rojas A. "Currículum oculto" en medicina: una reflexión docente. Rev Med Chile 2012; 140 (9): 1213-7.

3. Turbes S, Krebse E, Axtell S. The hidden curriculum in multicultural medical education: the role of case examples. Acad Med 2002; 77: 209-16.

4. Cribb A, Bignold S. Towards the reflexive medical school: the hidden curriculum and medical education research. Stud Higher Educ 1999; 24: 195-209.

5. Mossop L, Dennick R, Robbe I. Analysing the hidden curriculum: Use of a cultural web. Medical Education 2013; 47: 134-43.

6. Hafferty F. Beyond curriculum reform: confronting medicine`s hidden curriculum. Acad Med 1998; 73: 4037.

7. Haidet P, Kelly A, Chou C. Characterizing the patientcenteredness of hidden curricula in medical schools: development and validation of a new measure. Acad Med 2005; 80: 44-50.

8. Bitran M, Zúñiga D, Leiva I, Calderón M, Tomicic A, Padilla $\mathrm{O}$, et al. ¿Cómo aprenden los estudiantes de medicina en la transición hacia el ciclo clínico? Estudio cualitativo de las percepciones de estudiantes y docentes acerca del aprendizaje inicial de la clínica. Rev Med Chile 2014; 142 (6): 723-31.

9. Simons H. El estudio de caso: Teoría y práctica. Ediciones Morata. Madrid; 2011.

10. Vieytes R. Metodología de la investigación en organizaciones, mercado y sociedad. Editorial de las Ciencias. Buenos Aires; 2004.

11. Lempp H, Seale C. The hidden curriculum in undergraduate medical education: qualitative study of medical perceptions of teaching. BMJ 2004; 329: 770-3. 
12. Bardín L. Análisis de contenido. Ediciones Aka. Madrid; 1986.

13. Berelson B. Content Analysis. En Lindzey: Handbook of social psychology. Tomo I. New York; 1967.

14. Phillips A, Clarke M. More than an education: the hidden curriculum, professional attitudes and career choice. Acad Med 2012; 46: 887-93.

15. Gaufberg E, Batalden M, Sands R, Bell S. The hidden curriculum: what can we learn from third-year medical student narrative reflections? Acad Med 2010; 85: 170916.
16. Thiedke C, Blue A, Chessman A, Keller A, Mallin R. Student Observations and Ratings of Preceptor's Interactions with Patients: The Hidden Curriculum. Teaching and Learning in Medicine 2013; 16 (4): 312-6.

17. Wong A, Trollope-Kumar K. Reflections: an inquiry into medical student's professional identity formation. Med Educ 2014; 48: 489-501.

18. Neumann M, Edelhäuser F, Tauschel D, Fischer M, Wirtz $\mathrm{M}$, Woopen $\mathrm{C}$, et al. Empathy decline and its reasons: a systematic review of studies with medical students and residents. Acad Med 2011; 86: 996-1009. 